\title{
The Applicability of Four Grit Scales to a Traditional Collectivist Context
}

\author{
Chengchieh Li ${ }^{1, *}$ Hungchang Lee ${ }^{1}$
}

\author{
${ }^{1}$ School of Education, Zhaoqing University, Zhaoqing, Guangdong, China \\ *Corresponding author. Email: 1010765988@qq.com
}

\begin{abstract}
This study aims to examine four kinds of grit scales for 385 Chinese undergraduates using both withinnetwork and between-network approaches to construct validation. Our results revealed that grit was more suitable for a two-factor model than a hierarchical construct. Second, though the three-factor grit was the best fit for the Chinese context, the association between perseverance of effort and adaptability to situations may be conceptually problematic. Third, academic grit was more salient in predicting academic outcomes while adaptability to situations was more linked to well-being outcomes.
\end{abstract}

\section{Keywords: academic self-efficacy, grit, life satisfaction, subjective well-being}

\section{INTRODUCTION}

Grit was defined as the persistency of effort and the consistency of interests for long-term goals and was introduced to describe an individual ability to pursuit and maintain their passion with sustained effort [1]. Duckworth [1] conceptualized grit as a domain-general personality trait composed of two dimensions: perseverance of effort and consistency of interests. Perseverance of effort refers to the tendency to strive harder to accomplish goals in spite of difficulties or adversities while consistency of interests pertains to the inclination to espouse steadily the passion over time.

Initially, Duckworth [1] developed the original grit scale (Grit-O) and hoped it was suitable for a variety of populations and different domains, and it could offer salient psychometric evidences and meet a precise fit with the construct of grit. Afterwards, because Duckworth and Quinn [2] argued that Grit-O had poor psychometric properties, they extracted from it to a brief short grit scale (Grit-S). They indicated that the hierarchical model of grit had better fit indices compares to a two-factor model and suggested that the total score of Grit-S was a better predicator of more difficult and arduous task than was either factor alone.

With more attention on grit, some empirical studies raised questions about the construct of grit, the differentiation of overall score grit and two facets score of grit, and the application to non-Western context [3]. First, when it comes to the differentiation of grit score, some studies used the total score of grit while others adopted the two separate facets to represent grit. It remains unclear how these two ways might be differently related to various consequences. Duckworth and Quinn [2] argued that the total score of Grit-S has better predictability, but most studies shown that perseverance of effort has been more powerful predicted outcomes than consistency of interests when analyzed separately. What's more, only perseverance of effort is a strong positive predictor accounted for academic-related outcomes while consistency of interests had nothing to do with them [4]. Clark and Malecki [5] supposed that the predictive power of perseverance of effort is very likely to be dispersed by consistency of interests when combined into a total score of grit, so they developed a new grit scale for academic domain, called Academic Grit Scale (AGS).

Second, in terms of the construct of grit, some studies supported the two-factor model of grit instead of the hierarchical model of grit. Credé [3] indicated that a hierarchical structure of grit failed to be supported by meta-analysis, and evidenced to support a distinct twofactor structure of grit. Datu, Valdez, and King [6] concluded that grit couldn't be conceptualized as a hierarchical construct. In addition, we came upon that the factor loading of consistency of interests in a higher-order was lower than criterion value, and even the correlation coefficient between two factors of grit was low and non-significant in several studies. On the contrast, a few studies shown that the higher-order model of grit had more perfect factor loadings and model fit indices. There is still controversy over what the construct of grit could be two-factor model or a hierarchical model of grit.

Third, with regard to the application to collectivist societies, Datu [6] argued that Grit-S has been primarily suitable for individualism context, so it may limit the generalizability of result, especially in collectivist cultures. They found that the higher-order structure of grit wasn't confirmed with Filipino students, and 
consistency of interests may be disadvantageous in collectivist settings [7]. Datu [7] mentioned that it is likely that people in collectivist context have an inclination to establish and maintain harmonious relationship with significant others, so they don't need to endorse consistent interests for long-term goals, and their interests may change across time depending upon parents' or teachers' expectations. As a result, consistency of interests may not be associated with adaptive outcomes in collectivist or interdependent societies. Afterwards, Datu, Yuen, and Chen [8] offered a dimension, adaptability to situations, based on Grit-S, called as Triarchic Model of Grit Scale (TMGS), which may be more appropriated for collectivist cultures. Adaptability to situations refers to an ability to adjust effectively flexible and challenging situations [8].

Nevertheless, some empirical studies provided evidences of the cross-cultural similarities and differences according to Duckworth [1] grit theory. Regardless of Chinese or Korean samples, they appeared the same problems as we proposed above. For example, Hwang, Lim, and $\mathrm{Ha}$ [9] indicated that consistency of interests wasn't associated with academic maladjustment for Korean female undergraduates. Lee [10] found that consistency of interests had nothing to do with academic performance for Chinese students. However, Li et al. [11] shown that the higher-order model of grit had more perfect factor loadings for Chinese high school students. These conflicts findings make it inappropriate to conclusion that the construct of grit was suitable for cross-cultural generalizability and general domains, and had salient psychometric evidences, and meet a precise hierarchical model fit.

The purposes of the present study are to examine the cross-cultural applicability of four kinds of grit scales, to test the constructs of four grit scales if consistent with a hierarchical model, and to assess the relations of four grit scales with both academic and social-psycho outcomes. We had two research questions: 1) Which is the grit construct fit for the Chinese context? 2) How is grit associated with academic and social-psycho outcomes? We hypothesized that our data will support a distinct twofactor model of grit rather than a hierarchical model of grit. TMGS will be fit for the Chinese context. AGS and perseverance of effort will more predict academic outcomes.

\section{METHOD}

\section{A. Participants}

There were 358 undergraduates from a public university in China who took the course of educational psychology. The average age of the participants was $19.62(\mathrm{SD}=1.07)$. There were 300 female and 58 male students. Because the participants majored in elementary education from school of education, the female numbers were larger then male.

\section{B. Measures}

Regarding to grit scales, we used four different grit scales, including Original Grit Scale (Grit-O; [1]), Short Grit Scale (Grit-S; [2]), Triarchic Model of Grit Scale (TMGS; [8]), and Academic Grit Scale (AGS; [5]). Grit-O is a 12-item instrument that is composed of two dimensions: consistency of interests (e.g., "My interests change from year to year.") and perseverance of effort (e.g., "I am diligent"). Grit-S is an 8-item brief version extracted from the Grit-O. TMGS is a 10 -item scale with dimensions of perseverance of effort, consistency of interests, and adaptability to situations (e.g., "I appreciate new opportunities that come into my life") for collectivist societies. AGS is a 10-item academicrelated grit scale that pays an emphasis on the pursuit of challenging long-term goals within the domain of education (e.g., "I push myself to do my personal best in school"). All items of four grit scales are rated on a 5-point Likert scale from $1=$ not at all like me to $5=$ very much like me.

In addition to three kinds of grit scales, four instruments were assessed to conduct between-network validity, including academic self-efficacy, academic self-assessment, life satisfaction, and subjective wellbeing. Academic self-efficacy was measured with the 5item from a subset of Pattern of Adaptive Learning Scale (PALS). An example PALS item is, "Even if the work is hard, I can learn it". Participants rated items on a 5-point Likert-type scale ranging from 1 (not at all true) to 5 (very true). Academic self-assessment was measured by participants assessed themselves academic performance compared to classmates and peers, and rated items on a 5-point Likert-type scale ranging from 1 (the worst) to 5 (the best). A mean score was calculated with higher scores indicating better academic performance. Life satisfaction was measured with the 6item Mental Health Scale (MHS). An example MHS item is, "You feel your family is". Items are rated on a 5-point Likert-type scale from 1 (Strongly dissatisfied) to 5 (Strongly satisfied). A mean score was calculated with higher scores indicating greater life satisfaction. Subjective well-being is a one-item self-reported index which is rated on a 10-poit Likert-type scale. Higher score on this scale would indicate greater subjective well-being.

\section{Analysis}

We conducted within-network and between-network approaches to construct validation approach [12]. For the within-network validity, we used confirmatory factor analysis (CFA) to examine the psychometric validity and to see which one would yield the best model fit. The first model is a two-factor construct of 
Grit-O underpinned by perseverance of effort and consistency of interests (Model 1). Model 2 proposes the hierarchical model of Grit-O. Model 3 is a twofactor construct of Grit-S. Model 4 is a higher-order construct of Grit-S. Model 5 is a three-factor construct of TMGS based on perseverance of effort, consistency of interests and adaptation to situations. Model 6 proposes the hierarchical model of TMGS. Model 7 is a unidimensional model of AGS wherein all the items load onto a single latent academic grit factor. In terms of between-network validity, we investigated how grit was associated with academic self-efficacy, academic self-assessment, life satisfaction, and subjective wellbeing.

\section{RESULTS}

\section{A. Within-network constrcut validity}

To examine the within-network validity of four kinds of grit scales, we conducted a series of CFA models to examine which one would yield the best fit by model competition using Mplus 8 with maximum likelihood estimation method. We used multiple indices to evaluate the goodness of model fit, such as comparative fit index (CFI), Tucker-Lewis index (TLI), root mean square error approximation (RMSEA), standardized root mean square residual (SRMR), and chi-square statistics.

As reported in "Table I", the bivariate correlations, descriptive statistics, and internal consistency coefficients for all study variables were obtained. Kurtosis ranged from -0.34 to 1.03 , while skewness ranged from -0.60 to 0.02 , that don't exceed 7 and 2 respectively are considered normal distribution. The association of consistency of interests with perseverance of effort was small $(r=.16-.26)$, suggesting that psychometric strength of grit in adolescent populations and collectivist context may be questionable. Consistency of interests wasn't linked to academic self-efficacy, life satisfaction, and subjective well-being.

TABLE I. BIVARIATE CORRELATIONS, DESCRIPTIVE STATISTICS AND INTERNAL CONSISTENCIES FOR STUDY VARIABLES.

\begin{tabular}{|c|c|c|c|c|c|c|c|c|c|c|c|c|c|c|}
\hline Variable & 1 & 2 & 3 & 4 & 5 & 6 & 7 & 8 & 9 & 10 & 11 & $\mathbf{M}$ & SD & alpha \\
\hline 1.CI-O & & & & & & & & & & & & 3.27 & 0.66 & .77 \\
\hline 2.CI-S & $94 * * *$ & & & & & & & & & & & 3.26 & 0.69 & .71 \\
\hline 3.CI-T & $90 * * *$ & $94 * * *$ & & & & & & & & & & 3.23 & 0.69 & .64 \\
\hline 4.PE-O & $23 * * *$ & $26 * * *$ & $18^{* * *}$ & & & & & & & & & 3.23 & 0.68 & .78 \\
\hline 5.PE-S & $21 * * *$ & $23 * * *$ & $16^{* * *}$ & $.93 * * *$ & & & & & & & & 3.27 & 0.76 & .80 \\
\hline 6.PE-T & $23 * * *$ & $25 * * *$ & $17 * *$ & $.91 * * *$ & $95 * * *$ & & & & & & & 3.35 & 0.82 & .82 \\
\hline 7.AS & $11 *$ & $15 * *$ & .08 & $.66 * * *$ & $65 * * *$ & $63 * * *$ & & & & & & 3.66 & 0.70 & .77 \\
\hline 8.AG & $13 *$ & $15 * *$ & .09 & $.67 * * *$ & $65 * * *$ & $69 * * *$ & $64 * * *$ & & & & & 3.54 & 0.69 & .91 \\
\hline 9.AA & $17 * *$ & $18 * * *$ & $.13^{*}$ & $25 * * *$ & $26 * * *$ & $28 * * *$ & $27 * * *$ & $34 * * *$ & & & & 3.30 & 0.58 & 86 \\
\hline 10.ASE & $.11^{*}$ & $12 *$ & .07 & $44 * * *$ & $42 * * *$ & $43^{* * *}$ & $45 * * *$ & $.56 * * *$ & $28 * * *$ & & & 3.38 & 0.77 & .91 \\
\hline 11.LS & .01 & .06 & .02 & $38 * * *$ & $36 * * *$ & $32 * * *$ & $39 * * *$ & $39 * * *$ & $21 * * *$ & $40 * * *$ & & 3.43 & 0.66 & 81 \\
\hline 12.SWB & -.01 & .01 & -.03 & $24 * * *$ & $22 * * *$ & $18 * * *$ & $26 * * *$ & $22 * * *$ & $23 * * *$ & $24 * * *$ & $40 * * *$ & 6.65 & 1.52 & - \\
\hline
\end{tabular}

a. Note. $\mathrm{CI}=$ consistency of interests; $\mathrm{PE}=$ perseverance of effort; AS = adaptability to situations; $\mathrm{AG}=$ academic grit; $\mathrm{AA}=$ academic self-assessment; ASE = academic self-efficacy; $\mathrm{LS}=$ life satisfaction; SWB $=$ subjective well-being

The two-factor model of Grit-O (Model 1) had poor fit indices. The association of consistency of interests with perseverance of effort was small $(r=.289)$, and most of factor loadings of manifest variables were smaller than .70. The hierarchical model of Grit-O (Model 2) didn't be identified. The two-factor model of Grit-S (Model 3) had better fit indices than Model 1. The correlation between consistency of interests and perseverance of effort was still small $(r=.296)$, and some of factor loadings of manifest variables were smaller than .70, especially consistency of interests. The hierarchical model of Grit-S (Model 4) didn't still be identified. The three-factor model of TMGS (Model 5) had better fit indices than Model 3. This is not surprising that consistency of interests and perseverance of effort seem to be distinct and weekly correlated with each other $(r=.179)$. Adaptability was significant high linked to perseverance of effort $(r=.764)$ while consistency of interests wasn't. The higher-order model of TMGS (Model 6) had the same fit indices as Model
5. However, the latent variable covariance matrix between perseverance of effort and adaptability to situations wasn't positive definite, this could indicate a linear dependency. Finally, the unidimensional model of AGS (Model 7) had better fit indices than Model 1 and Model 3, but it had a few worse than Model 5. Therefore, the three-factor model of TMGS and AGS were chosen as the final model and were applicable for Chinese collectivist society. These results were shown in "Table II". 
TABLE II. MODEL FIT INDICES OF THE HYPOTHESIZED AND ALTERNATIVE MODELS OF GRIT.

\begin{tabular}{|c|c|c|c|c|c|c|c|c|c|}
\hline Model & $\chi^{2}$ & df & $\chi^{2} / \mathbf{d f}$ & CFI & TLI & RMSEA & SRMR & AIC & BIC \\
\hline 1 & $191.45 * * *$ & 53 & 3.612 & .890 & .863 & .085 & .069 & 10831.44 & 10975.02 \\
\hline 3 & $74.16^{* * * *}$ & 19 & 3.903 & .933 & .901 & .090 & .062 & 7142.21 & 7239.22 \\
\hline 5 & $83.13 * * *$ & 32 & 2.598 & .957 & .939 & .067 & .056 & 8456.75 & 8584.80 \\
\hline 6 & $83.13 * * *$ & 32 & 2.598 & .957 & .939 & .067 & .056 & 8456.75 & 8584.80 \\
\hline 7 & $146.18 * * *$ & 35 & 4.176 & .938 & .921 & .094 & .039 & 8004.71 & 8121.13 \\
\hline
\end{tabular}

\section{B. Between-network construct validity}

To examine the between-network construct validity of four kinds of grit scales, they were entered as predictors of academic self-assessment, academic selfefficacy, life satisfaction, and subjective well-being. In terms of Grit-O, perseverance of effort positively predicted academic and well-being outcomes while consistency of interests didn't significantly predict all outcomes $\left(\chi^{2}=653.86, \mathrm{df}=336, \mathrm{CFI}=.895\right.$, TLI $=$ .882 , RMSEA $=.051$, SRMR $=.060$ ). In the light of Grit-S, perseverance of effort still positively predicted academic and well-being outcomes. Consistency of interests positively predicted academic self-assessment while it didn't significantly predict the other outcomes $\left(\chi^{2}=458.45, \mathrm{df}=238, \mathrm{CFI}=.915, \mathrm{TLI}=.901, \mathrm{RMSEA}\right.$ $=.051$, SRMR $=.058)$. With respect to TMGS, consistency of interests still positively predicted academic self-assessment, but it didn't significantly predict the other outcomes. Adaptability to situations positively predicted academic and well-being outcomes; however, it is surprising that perseverance of effort didn't significantly predict all outcomes $\left(\chi^{2}=\right.$ $532.10, \mathrm{df}=279, \mathrm{CFI}=.916, \mathrm{TLI}=.902, \mathrm{RMSEA}=$ .050, SRMR = .056). Finally, academic grit significantly predicted academic self-assessment, academic self-efficacy, life satisfaction, and subjective well-being $\left(\chi^{2}=595.42, \mathrm{df}=290, \mathrm{CFI}=.916\right.$, TLI $=$ .906$, RMSEA $=.054$, SRMR $=.060)$.

TABLE III. PATH ANALYSES OF FOUR GRIT SCALES AS PREDICTORS OF ACADEMIC AND WELL-BEING OUTCOMES.

\begin{tabular}{|c|c|c|c|c|}
\hline Variable & Academic self-assessment & Academic self-efficacy & Life satisfaction & Subjective well-being \\
\hline Consistency-O & $.108(.066)$ & $.012(.060)$ & $-.034(.076)$ & $-.077(.062)$ \\
\hline Perseverance-O & $292 * * *(.060)$ & $478 * * *(.052)$ & $.277 * * *(.071)$ & $.230 * * *(.058)$ \\
\hline$R^{2}$ & $115^{* *}$ & $232 * * *$ & $.072 *$ & $.049 *$ \\
\hline Consistency-S & $.166^{*}(.068)$ & $.048(.065)$ & $.022(.081)$ & $-.033(.066)$ \\
\hline Perseverance-S & $.275 * * *(.060)$ & $446^{* * *}(.054)$ & $.234 * *(.072)$ & $.202 * *(.059)$ \\
\hline$R^{2}$ & $130 * *$ & $213 * * *$ & .058 & .038 \\
\hline Consistency-T & $.160 *(.068)$ & $.062(.064)$ & $.023(.080)$ & $-.024(.068)$ \\
\hline Perseverance-T & $.118(.112)$ & $.108(.105)$ & $-.035(.133)$ & $-.100(.108)$ \\
\hline Adaptability-T & $.234 *(.234)$ & $440 * * *(.104)$ & $.346^{*}(.136)$ & $.374 *(.108)$ \\
\hline$R^{2}$ & $152 * * *$ & $290 * * *$ & $104 *$ & $.092 *$ \\
\hline Academic grit & $.384 * * *(.052)$ & $607 * * *(.039)$ & $268 * * *(.067)$ & $.230 * * *(.052)$ \\
\hline$R^{2}$ & $147 * * *$ & $369 * * *$ & $.072 *$ & $.053 *$ \\
\hline
\end{tabular}

As reported in "Table III", for academic selfassessment, TMGS and AGS were significant and accounted for $15.2 \%$ and $14.7 \%$ respectively. Although the accounting for variance of TMGS was higher than AGS', it is interesting that perseverance of effort wasn't associated with academic self-assessment. Therefore, academic grit was the best significant predictor of academic self-assessment $(\gamma=.384)$ of the other variables. With regard to academic self-efficacy, academic grit was significant and accounted for $36.9 \%$ and was the best significant predictor of academic selfefficacy $(\gamma=.607)$. Moreover, perseverance of effort was associated with academic outcomes regardless of Grit-O and Grit-S while consistency of interests wasn't linked to academic self-efficacy. These results provide evidence of academic grit in accounting for variance in academic outcomes beyond the others.

When it came to well-being outcomes, the accounting for variance of TMGS was higher than
AGS, but perseverance of effort in TMGS didn't significantly predict life satisfaction and subjective well-being. Interestingly, adaptability to situations was the best significant predictor of life satisfaction $(\gamma=$ $.346)$ and subjective well-being $(\gamma=.374)$. Moreover, perseverance of effort for Grit-O and Grit-S was associated with well-being outcomes, but consistency of interests wasn't. These results provide evidence of academic grit in accounting for variance in well-being outcomes beyond the others.

\section{CONCLUSION}

The first purpose of this study was to test the factor structure of grit in China taking regard as a traditional collectivist context. The results of within-network validation didn't support the hierarchical structure of grit as proposed by Duckworth [1]. This suggests that grit was more suitable for a two-factor model, and it is appropriate to adopt two separate dimensional scores in 
future research. It is notable that the correlation between perseverance of effort and consistency of interests was very week. It is obvious that Duckworth's grit theory may be conceptually problematic.

We demonstrated that the three-factor of TMGS was an acceptable measurement model of grit in Chinese collectivist society. Precisely, we found that adaptability to situations was far higher associated with perseverance of effort while it wasn't linked to consistency of interest at all. It seems that adaptability to situations overlaps with perseverance of effort though Datu [8] argued that they could serve as interlocking dimensions. They explained that collectivists may struggle to maintain more diligence to cope with the challenging circumstances or to adapt to various expectations from significant others. Doubtfully, the result of their study shown that adaptability to situations and perseverance of effort were both associated with psychological outcomes, but we found that adaptability to situations was the only salient in predicting academic and well-being outcomes rather than perseverance of effort. This finding suggests that the psychometric strength for TMGS may be questionable in our populations.

We suggest that the single-factor structure of academic grit was supported and may be considered acceptable in Chinese collectivist context. In addition, not only may academic grit be ideal to investigate academic-specific outcomes, but also it had the same predictability to well-being outcomes as perseverance of effort. It is crucial that future research investigate the validation and applicability of TMGS for other collectivist cultures, assess academic grit's stability, and ascertain the psychometric adequacy of academic grit.

\section{References}

[1] Duckworth, A. L., Peter, C., Matthews, M. D., and Kelly, D. R. "Grit: Perseverance and passion for long-term goals," Journal of Personality and Social Psychology, vol. 92, pp. 1087-1101, 2007.

[2] Duckworth, A. L., and Quinn, P. D. "Development and validation of the short grit scale (Grit-S)," Journal of Personality Assessment, vol. 91, pp. 166-174, 2009.

[3] Credé, M., Tynan, M. C., and Harms, P. D. "Much ado about grit: A meta-analytic synthesis of the grit literature," Journal of Personality and Social Psychology, vol. 113, pp. 492-511, 2017.

[4] Wolters, C. A., and Hussain, M. "Investigating grit and its relations with college students' self-regulated learning and academic achievement," Metacognition Learning, vol. 10, pp. 293-311, 2015.

[5] Clark, K. N., and Malecki, C. K. "Academic grit scale: Psychometric properties and associations with achievement and life satisfaction," Journal of School Psychology, vol. 72, pp. 4966, 2019.

[6] Datu, J. A. D., Valdez, J. P. M., and King, R. B. "Perseverance counts but consistency does not! Validating the short grit scale in a collectivist setting," Current Psychology, Vol. 35, pp. 121 130, 2016.

[7] Datu, J. A. D., Yuen, M., and Chen, G. "Exploring determination for long-term goals in a collectivist context: A qualitative study," Current Psychology, vol. 37, pp. 263-271, 2018.

[8] Datu, J. A. D., Yuen, M., and Chen, G. "Development and validation of the triarchic model of grit scale (TMGS): Evidence from Filipino undergraduate students," Personality and Individual Differences, vol. 114, pp. 198-205, 2017.

[9] Hwang, M. H., Lim, H. J., and Ha, H. S. "Effects of grit on the academic success of adult female students at Korean open university," Psychological Reports, vol. 121, pp. 705-725, 2018.

[10] Lee, W. W. S. "Relationships among grit, academic performance, perceived academic failure, and stress in associate degree students," Journal of Adolescence, vol. 60, pp. 148-152 2017.

[11] Li, J., Zhao, Y., Kong, F., Du, S., Yang, S., and Wang, S. "Psychometric assessment of the short grit scale among Chinese adolescents," Journal of Psychoeducational Assessment, vol. 36 pp. 291-296, 2018

[12] Marsh, H. W. "The measurement of physical self-concept: A construct validation approach," in The physical self: From motivation to well-being, K. Fox, Ed. Champaign, IL: Human Kinetics, 1997, pp.27-58. 